\title{
Vector Meson and Associated Strangeness Production Using a Linearly Polarized Photon Beam at Jefferson Lab
}

\author{
Philip L. Cole \\ Jefferson Lab, 12000 Jefferson Ave., Newport News, VA 23606, USA \\ (the CLAS Collaboration: C. Gordon, J. Kellie, F. Klein, K. Livingston, S. Mehrabyan, J. Melone, \\ J.C. Sanabria, D. Tedeschi, and M. Wood)
}

Received on 4 November, 2003

\begin{abstract}
The set of experiments forming the g8a run took place in the summer of 2001 in Hall B of Jefferson Lab. The g8a run was the commissioning experiment for the linearly-polarized photon beam at CLAS. The aim of these experiments is to improve the understanding of the underlying symmetry of the quark degrees of freedom in the nucleon, the nature of the parity exchange between the incident photon and the target nucleon, and the mechanism of associated strangeness production in electromagnetic reactions. A beam of tagged and collimated linearly polarized photons (energy range 1.8-2.2 GeV) in conjunction with the large solid angle coverage of CLAS make possible the extraction of the differential cross-sections and polarization observables for the photoproduction of vector mesons and kaons. The reaction channels $\vec{\gamma} \mathrm{p} \rightarrow \rho^{0} \mathrm{p} \rightarrow \pi^{+} \pi^{-} \mathrm{p}$ and $\vec{\gamma} \mathrm{p} \rightarrow \mathrm{K}^{+} \Lambda^{0} \rightarrow \mathrm{K}^{+} \pi^{-} \mathrm{p}$ are under investigation to search for possibly missing nucleon resonances. An overview of the experiment and preliminary results on the measurement of the photon asymmetries of the aforementioned reactions will be presented in this paper.
\end{abstract}

\section{Beamline for producing linearly polarized photons}

The g8 group of experiments [1-4] employed the coherent bremsstrahlung facility, which was commissioned at the beginning of the g8a run in Hall B of JLab [5, 6] (see Fig. 1). To produce polarized photons of energy $2.0 \mathrm{GeV}$ from an incident $5.7-\mathrm{GeV}$ electron beam on a diamond radiator requires that the angle of incidence between the reciprocal lattice vector of the radiator and the electron beam be aligned to approximately $1 \mu \mathrm{rad}$. We employed the GWU goniometer [7] to align a 50- $\mu \mathrm{m}$ diamond radiator. The technique of Glasgow University for aligning the crystal by means of a series of scans is an extension of Lohman [8], and will be detailed in an upcoming NIM article [9].

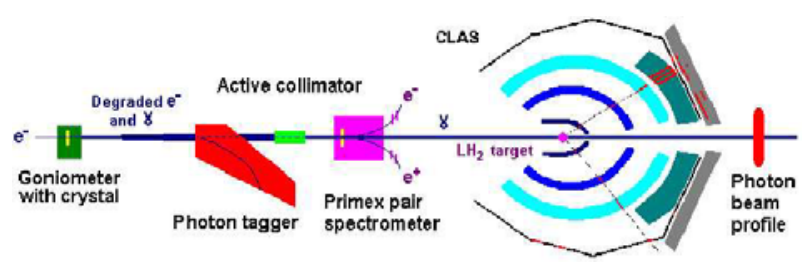

Figure 1. The Hall B beamline for the coherent bremsstrahlung facility at Jefferson Lab.

The alignment procedure entails executing small angular movements of the crystal and recording the corresponding photon tagger spectrum for each shift in $\theta_{\mathrm{h}}$ and $\theta_{\mathrm{v}}$. The [100] crystal axis is set at $60 \mathrm{mrad}$ from its nominal position and is swept through a $360^{\circ}$ cone in azimuth. The procedure for aligning the crystal is shown in Fig. 2, where on the left we show a simulation of a scan that is not yet aligned; the $\theta_{h}-\theta_{v}$ offset indicates the degree that the [100] plane is not perpendicular to the electron beam direction. The scan on the right is real data. The dark radial ridges of these plots trace the energy of the coherent peak as the angle between the beam and the face of the crystal varies. As can be seen in the left hand figure, the orientation of the crystal with respect to the beam is found by fitting a template (shown with dashed lines) composed of 8 lines, spaced $45^{\circ}$ apart. The final scan is close to a perfect 4-fold symmetry, showing that the crystal is very well aligned to the beam, as indicated in the right-hand panel in Fig. 2. The photon spectrum, as measured by the photon tagger [10], is the key diagnostic tool for aligning the reciprocal lattice vectors of the diamond radiator with respect to the incident electron beam to allow for coherent bremsstrahlung production. From the scan plot on the right in Fig. 2 (see also Fig. 3), we can see that the photon tagger performed very well.

An instrumented collimator [11] of aperture $2 \mathrm{~mm}$ was installed in the Hall-B beamline downstream of the tagger magnet at a distance of $22.9 \mathrm{~m}$ from the diamond radiator. The collimator was sensitive to beam shifts to better than $25 \mu \mathrm{m}$.

\section{Production running of g8a}

The g8a production running period took place in the summer of 2001 (July 12 - August 13). The energy of the incident electron beam on the 50- $\mu \mathrm{m}$ thick diamond radiator was $5.7 \mathrm{GeV}$, with a nominal current of $7 \mathrm{nA}$. For our coherent bremsstrahlung data, we ran at three separate coherent 

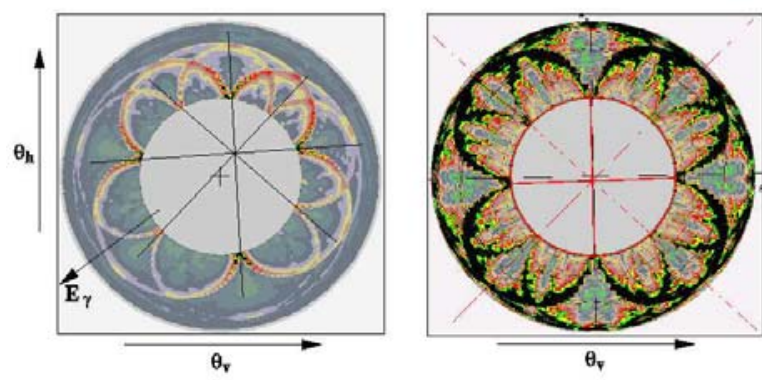

Figure 2. (Left) Simulated scan illustrating the alignment procedure by fitting a template. Here the face of the crystal, i.e. the [100] plane, is offset in $\theta_{\mathrm{v}}$ and $\theta_{\mathrm{h}}$ with respect to the direction of the incident electron beam. (Right) Final scan taken during a data scan run. We see that the crystal is aligned to the desired degree of precision.

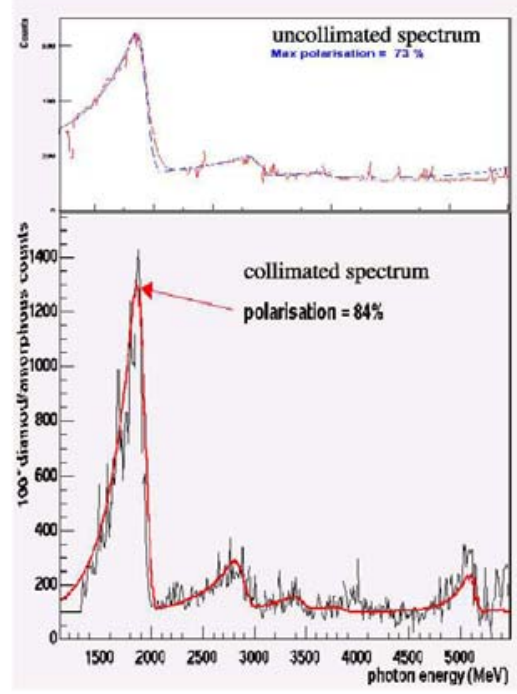

Figure 3. Normalized photon spectra before and after collimation. We remark that both plots have the same vertical scale; this highlights the enhancement of the spectral peak due to the collimation of the photon beam. The fits to both the uncollimated and collimated normalized photon spectra are from the anb code [12].

peak edge energies: $1.8 \mathrm{GeV}, 2.0 \mathrm{GeV}$, and $2.2 \mathrm{GeV}$. For understanding and delineating the systematics of the azimuthal dependence of the CLAS detector, we rotated the photonbeam polarization axis by $90^{\circ}$ on several occasions. We did this by periodically rotating the diamond crystal between the Miller indices of [02 $\overline{2}$ ] and [022], so that these two mutually perpendicular reciprocal lattice vectors were properly aligned with respect to the incident $5.7 \mathrm{GeV}$ electron beam. To further eliminate misleading or 'built-in' azimuthal dependences of the CLAS detector, we took several unpolarized photon runs employing the amorphous $50-\mu$ m thick carbon radiator in lieu of the diamond crystal. These incoherent bremsstrahlung data will furthermore be our yardsticks for determining the polarization of the beam. These complementary data sets will aid us in understanding our azimuthal dependences and thereby will serve to reduce the systematic uncertainties in the differential cross sections of the hyperons and vector mesons.
In Fig. 3 we plot the normalized photon spectrum before and after the collimator. Here, normalized means that the spectrum obtained with the diamond radiator is divided by the reference spectrum from an amorphous carbon radiator of thickness $50 \mu \mathrm{m}$, and where we have set the baseline to the value of 100 . The uncollimated data were collected online with the free-running scalers. The collimated spectrum is derived from TDC hit patterns with the random background subtracted out. This preliminary spectrum results from a first pass through the data with a rough timing calibration. Both data sets, i.e. uncollimated and collimated, are fit with the anb code [12]. We see excellent agreement between the data and the fits, and based upon this calculational evidence, we claim we have succeeded in producing a beam of linearly-polarized photons with a high degree of linear polarization of up to a maximum of $84 \%$.

\section{Analysis of the reaction channel $\vec{\gamma} \mathrm{p} \rightarrow \rho^{0} \mathrm{p} \rightarrow \pi^{+} \pi^{-} \mathrm{p}$}

The primary decay branch of the $\rho^{0}$ is to $\pi^{+} \pi^{-}(\sim 100 \%)$, the mass is $770 \mathrm{MeV}$, and the width $\Gamma=150 \mathrm{MeV}$. Exclusive $\vec{\gamma} p \rightarrow p \pi^{+} \pi^{-}$reactions are obtained by detecting all three outgoing particles in CLAS, and applying the following cuts: |missing energy $\mid \leq 0.04 \mathrm{GeV}$, $\mid$ missing transverse/longitudinal momentum $\mid \leq 0.04 \mathrm{GeV} / \mathrm{c}$, and $\mid$ missing mass ${ }^{2} \mid \leq 0.004 \mathrm{GeV} / \mathrm{c}^{2}$.

The main problem of identifying a clean $\rho^{0}$ signal is the contribution of $\pi^{+} \pi^{-}$from $\pi N^{*}, N^{*} \rightarrow \pi N$. Dalitz plots allow allow the $\rho^{0}$ signal to be emphasized in the forward direction i.e. low values of Mandelstam variable $|t|$ (4-momentum transfer squared to the proton). In such plots the (invariant mass) $)^{2}$ of the $\pi^{+} \pi^{-}$is plotted against that of the $p \pi^{+}$, and also against that of the $p \pi^{-}$, for all $|t|$. Fig. 4 shows the problem of isolating the $\rho^{0}$ more clearly. The high intensity signal in both plots for the (invariant mass) ${ }^{2}$ of the $\pi^{+} \pi^{-}$of $0.6 \mathrm{GeV}^{2}$ corresponds to the $\rho^{0}$. The horizontal bands passing through the vertical $\rho^{0}$ signal in both cases correspond to the overlapping resonances. The following (invariant mass) $)^{2}$ cuts have been applied to enhance the $\rho^{0}$ signal above these resonances: $0.4 \leq \pi^{+} \pi^{-} \leq 0.8 \mathrm{GeV}^{2}$, $1.8 \leq p \pi^{+} \leq 3.1 \mathrm{GeV}^{2}$, and $1.8 \leq p \pi^{-} \leq 3.1 \mathrm{GeV}^{2}$. For $|t|>0.45 \mathrm{GeV}^{2}$ clean $\rho^{0}$ identification is difficult. Analysis below this value is presented below.

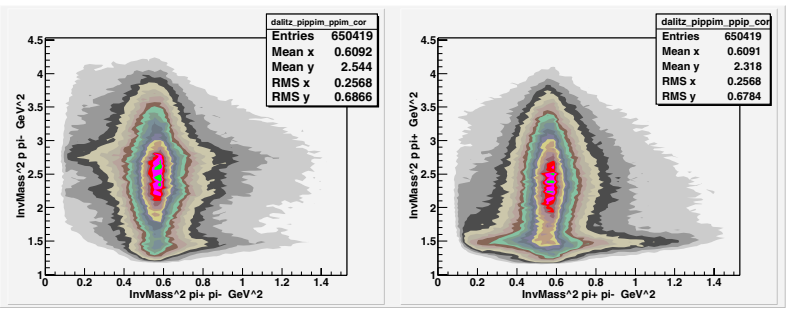

Figure 4. (Left) A dalitz plot showing the real $\rho^{0}$ signal as a vertical red band centered around $0.6 \mathrm{GeV}^{2}$, and overlapping resonances appearing as the horizontal bands from the detected $p \pi^{-}$particles. (Right) A similar dalitz plot as before, except the horizontal bands arise from a resonance from the detected $p \pi^{+}$particles. 
A typical definition of the photon asymmetry, $\Sigma$, is shown in eqn 1. Here $P_{\gamma}$ is the degree of linear polarization, and $Y_{\|}$and $Y_{\perp}$ are the yields of the $\rho^{0}$ to emerge in the plane of photon polarization and perpendicular to it. An alternative definition of $\Sigma$ can be obtained by integrating complete angular distribution, $W(\cos \theta, \phi, \Phi)$, with respect to $\theta$ and $\phi[15]$.

Here $\theta$ and $\phi$ are the polar and azimuthal angles of the $\pi^{+}$decay product in the helicity frame [1], $\Phi$ is the angle between the photon polarization vector and the production plane, $P_{\gamma}$ is the degree of linear polarization, and $\rho_{\alpha \beta}^{n}$ are the spin density matrix elements [1], as shown in eqn 2 . Here, the photon asymmetry parameter $\Sigma$ is obtained from measuring the azimuthal distribution of the production plane with respect to the angle of the photon electric polarization vector, $\Phi$, in the overall $(\vec{\gamma} p)$ c.m. system as depicted in Fig. 5. The large solid angle coverage of CLAS provides an excellent means for making this measurement.

$$
\begin{gathered}
\Sigma=\frac{1}{P_{\gamma}} \frac{Y_{\|}-Y_{\perp}}{Y_{\|}+Y_{\perp}} \\
W(\Phi)=1-\Sigma P_{\gamma} \cos 2 \Phi
\end{gathered}
$$

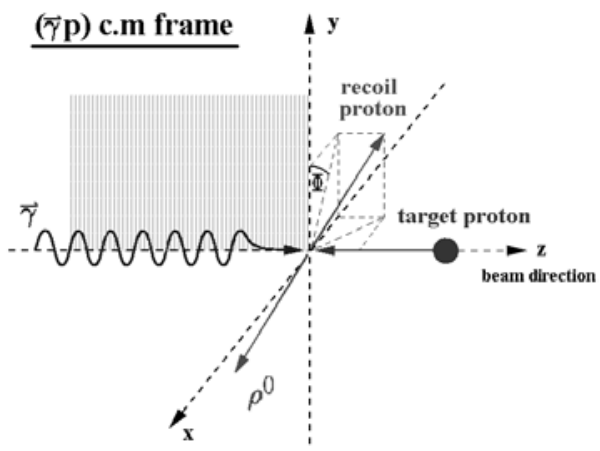

Figure 5. The $(\vec{\gamma} p)$ c.m. system. $\Phi$ is the azimuthal distribution of the production plane with respect to the angle of the photon electric polarization vector.

A selection of perpendicularly linearly polarized photon data taken with the coherent edge at $2.0 \mathrm{GeV}$ has been sampled to measure $\Phi$. This measurement allied with an estimate of the degree of linear polarization allows a first measurement of $\Sigma$ to be extracted using eqn. 2. The same event selection and kinematic cuts are also applied to a sample of unpolarized data obtained with an amorphous radiator, and $\Phi$ is measured for this data set also. To remove systematics and areas of non-fiducial acceptance, the $\Phi$ distribution from the polarized data is divided through by a scaled unpolarized distribution. The results of this method are shown in Fig. 6, and the corresponding asymmetries assuming an average polarization of $80 \%$ are presented in Table I. Fig. 7 is a first comparison with the quark model for baryons of Qiang Zhao [14]. This is a semi-empirical calculation with parameters based on the $\omega$. A coherent study of $\omega$ and $\rho$ is currently being worked on.

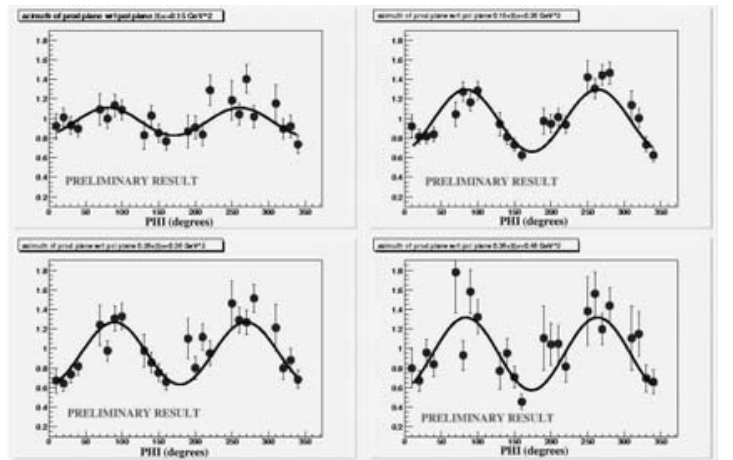

Figure 6. The measurement of $\Phi$ for four $-\mathrm{t}-$ bins with photon energy $E_{\vec{\gamma}}=2.0 \mathrm{GeV}$. Error bars shown are from statistical errors only.

TABLE I. Calculated asymmetry results for each $|t|$ bin with photon energy $E_{\vec{\gamma}}=2.0 \mathrm{GeV}$. The estimated polarization used is $80 \%$. Errors are calculated from statistical errors in the fitting of $\Phi$ and $5 \%$ for systematic errors.

\begin{tabular}{|c|c|}
\hline$|t|$ bin range $\mathrm{GeV}^{2}$ & $\Sigma=-\frac{\frac{W\left(0^{\circ}\right)-W\left(90^{\circ}\right)}{W\left(0^{\circ}\right)+W\left(90^{0}\right)}}{P_{\gamma}}$ \\
\hline \hline$|t| \leq 0.15$ & $0.183 \pm 0.036$ \\
\hline $0.15<|t| \leq 0.25$ & $0.406 \pm 0.025$ \\
\hline $0.25<|t| \leq 0.35$ & $0.418 \pm 0.033$ \\
\hline $0.35<|t| \leq 0.45$ & $0.492 \pm 0.046$ \\
\hline
\end{tabular}

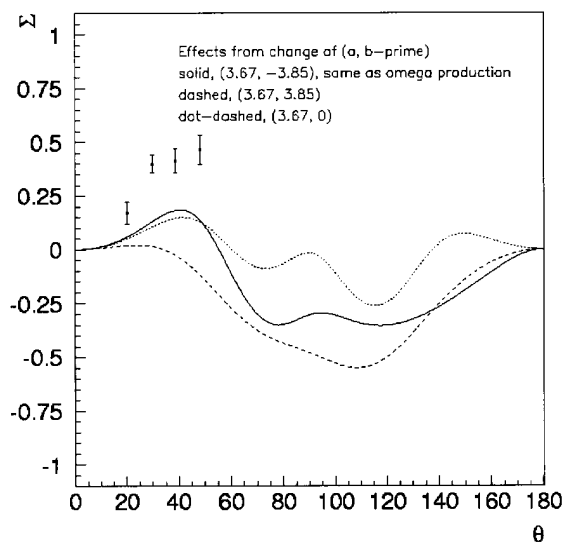

Figure 7. A first comparison with the quark model of baryons of Qiang Zhao [14]. This is a semi-empirical calculation uses parameters based on the $\omega$. A coherent study of $\omega$ and $\rho$ is currently being worked on.

\section{Analysis of the reaction channel $\vec{\gamma} \mathrm{p} \rightarrow \mathrm{K}^{+} \Lambda^{0} \rightarrow \mathrm{K}^{+} \pi^{-} \mathrm{p}$}

The decay of the $\Lambda^{0}(1115) \rightarrow \mathrm{p}+\pi^{-}$has a branching ratio of $\sim 64 \%$ and a mean lifetime of $\sim 2.6 \times 10^{-10}$ seconds. CLAS detects the $\mathrm{K}^{+}, \pi^{-}$and $\mathrm{p}$ directly, so the reaction is exclusive. The main problem of detecting this reaction is $\pi^{+}$contamination in the $\mathrm{K}^{+}$signal, which then brings nonexclusive reactions inside the cuts placed on the data, increasing the background. 


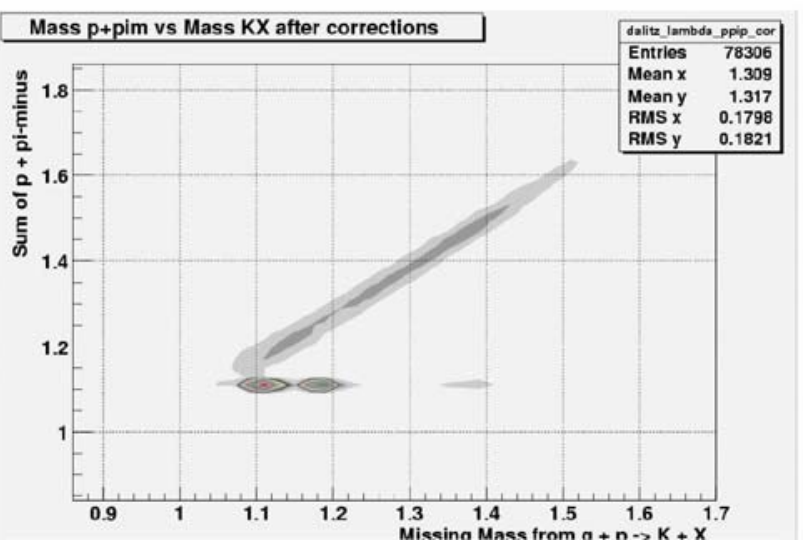

Figure 8. The relative kinematics of the $\mathrm{K}^{+}$and $\mathrm{p}+\pi^{-}$components of the overall reaction.

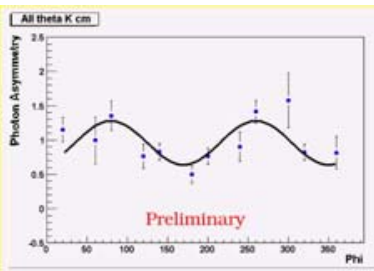

$$
40 \times \operatorname{tatakem}<60
$$
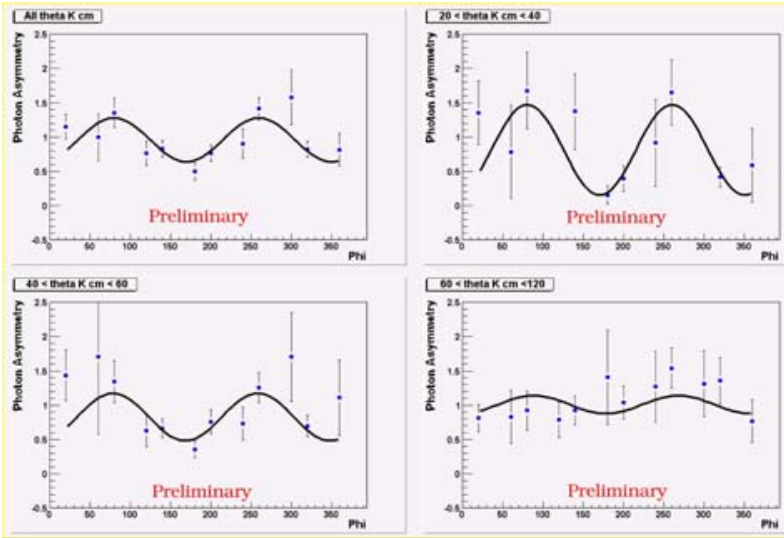

Figure 9. $\Phi$ results from each $\theta$ bin, with $E_{\vec{\gamma}}=2.25 \mathrm{GeV}$

Kinematic constraints can be used to reduce this effect, and by considering a plot of the missing mass of $\vec{\gamma}+\mathrm{p} \rightarrow \mathrm{K}^{+}+\mathrm{X}$ versus $\Lambda^{0} \rightarrow \mathrm{K}^{+}+\pi^{-}$, the background from misidentified kaons can be seen as a rising diagonal above the main kinematic region of the reaction. In Fig. 4 the $\Lambda^{0}$ peak is clearly identifiable and this enables a simple cut to be made around the peak, the parameters of the cut being $1.08 \mathrm{GeV}<$ Missing Mass $(\mathrm{X})<1.14 \mathrm{GeV}$ and the same parameters for Missing Mass $\left(\mathrm{p}+\pi^{-}\right)$.

As shown in eqn. 2 the photon asymmetry is calculated from particle yield over a range of azimuthal acceptance. Also as in Fig. 5, we calculate $\Phi$ with respect to the polarization vector of the incoming photon, in this case the $\mathrm{K}^{+}$and the $\Lambda^{0}$ are substituted for the $\rho$ and proton respectively. With the $\mathrm{K}^{+} \Lambda^{0}$ reaction well identified in CLAS, the azimuthal distribution is plotted over three $\theta$ bins defined by the $\mathrm{K}^{+}$ in the $(\vec{\gamma} \mathrm{p})$ c.m. frame. Each set of polarized data defined in this way is normalized by the corresponding amorphous data set, which reduces the effect of non-fiducial regions of CLAS and other systematics, as in Section 3. The resulting data sets are shown below in Fig. 9, where the azimuthal distribution is divided into $10^{\circ}$ bins to improve statistics for this preliminary calculation. The error bars come from the statistical weights of each point in the fit to the data.
TABLE II. Calculated asymmetry results for each $\theta_{C . M}^{K^{+}}$. bin, assuming a linear photon polarization of $\sim 80 \%$. Again, the systematics are $5 \%$.

\begin{tabular}{|c|c|}
\hline$\theta_{K}+$ c.m. & $\Sigma=-\frac{\frac{W\left(0^{\circ}\right)-W\left(90^{0}\right)}{W\left(0^{\circ}\right)+W\left(90^{0}\right)}}{P_{\gamma}}$ \\
\hline \hline $20<\theta<40$ & $0.87 \pm 0.29$ \\
\hline $40<\theta<60$ & $0.45 \pm 0.16$ \\
\hline $60<\theta<120$ & $0.17 \pm 0.16$ \\
\hline
\end{tabular}

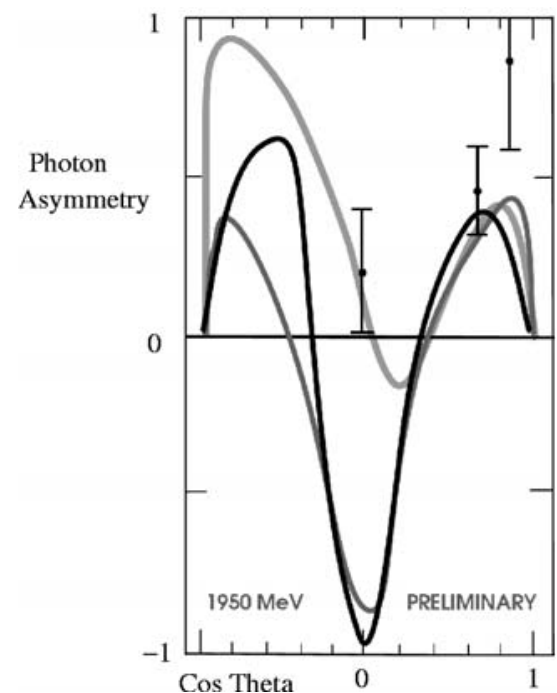

Figure 10. Preliminary comparison between the data and the theoretical calculations of Stijn Janssen made from fits to the SAPHIR data at $1.95 \mathrm{GeV}$. The relevance of the color scheme is discussed in the text.

Shown in Fig. 10 are plots of predicted photon asymmetry from the quark model calculations of Stijn Janssen [16]. This model makes calculations of the polarization observables of the $\mathrm{K}^{+} \Lambda^{0}$ reaction, which are variables which contribute to the differential cross section of this reaction when linearly-polarized photons are used [17].

$$
\begin{aligned}
\frac{d \sigma}{d \Omega} & =\sigma^{0}\left\{1-P_{b} \Sigma \cos 2 \phi-P_{x^{\prime}}\left(P_{b} O_{x^{\prime}} \sin 2 \phi\right)\right. \\
& \left.-\quad P_{y^{\prime}}\left(P_{b} T \cos 2 \phi\right)-P_{z^{\prime}}\left(P_{b} O_{z^{\prime}} \sin 2 \phi\right)\right\}(3)
\end{aligned}
$$

Janssen's model uses data from the SAPHIR [18] collaboration on the differential cross-section of $\mathrm{K}^{+} \Lambda^{0}$ to make estimates of the coupling strength $g_{K \Lambda p}$, which is an essential parameter for using elements of the Isobar model and Regge Trajectory model to make predictions of the polarization observables, in particular $\Sigma, \mathrm{P}_{\mathrm{x}}, \mathrm{P}_{\mathrm{y}}$, and $\mathrm{P}_{\mathrm{z}}$. Using the Isobar model allows a comparison to be made between the presence of different reactions or resonances in the reaction, and this alters the prediction of the photon asymmetry accordingly.

The predictions shown in Fig. 9 relate to three groups of resonances that Janssen includes in the model. The black line is a prediction of the photon asymmetry parameter $\Sigma$ at $E_{\vec{\gamma}}=1950 \mathrm{MeV}$, including the $N^{*}$ baryon resonances (S11(1650), P11(1710), P13(1720)).

The red line includes the "missing" resonance P11(1895), and the green line the "missing" D13(1895). 


\section{Summary}

With the g8a run, we were able to show 'proof of principle' that we can coherently produce a tagged and collimated beam of linearly-polarized photons from a 50- $\mu$ m diamond radiator. The maximum degree of polarization exceeds $80 \%$. For the first phase of g8, i.e. g8a, we collected approximately 1.8 billion triggers, which, after our data cuts and analysis, should give us over 100 times the world's data set for linearly polarized photoproduction of vector mesons in the energy range of $1.8 \leq E_{\vec{\gamma}} \leq 2.2 \mathrm{GeV}$. In summary, a linearly-polarized photon beam represents a real enhancement of the JLab facility and its physics capabilities.

\section{Acknowledgments}

P.L. Cole thanks Prof. Roberto Ribas of the University of São Paulo for his work as a coPI on the NSF Grant Award INT-0313656, which provided funding to sponsor nine students to attend this conference. Cole furthermore compliments Dr. Ribas for organizing a successful and thoughtprovoking conference. This work is supported through grants from the U.S. Department of Energy, the National Science Foundation, and the Engineering and Physical Sciences Research Council of the U.K.

\section{References}

[1] "Photoproduction of $\rho$ Mesons from the Proton with Linearly Polarized Photons," JLab E94-109, P.L. Cole, J.P. Connelly, and K. Livingston, cospokespersons.

[2] "Photoproduction of $\phi$ Mesons with Linearly Polarized Photons," JLab E98-109, D.J. Tedeschi, P.L. Cole, and J.A. Mueller, cospokespersons.

[3] "Photoproduction of $\omega$ Mesons off Protons with Linearly Polarized Photons," JLab E99-013, F.J. Klein and P.L. Cole, cospokespersons.
[4] "Photoproduction of Associated Strangeness using a Linearly Polarized Beam of Photons," CLAS Approved Analysis 2001-02, J.C. Sanabria, J. Kellie, and F.J. Klein, cospokespersons.

[5] Jefferson Lab, Virginia, USA, http://www.jlab.org.

[6] B.A. Mecking, et al., Nucl. Instr. and Meth. 503/3, 513 (2003).

[7] NSF Award 9724489, W.J. Briscoe, K.S. Dhuga, and L.C. Maximon, co-PIs, The George Washington University, Washington, D.C., 20052.

[8] D. Lohman, M. Schumacher et al., Nucl. Instr. and Meth. A 343, 494 (1994).

[9] K. Livingston, to be submitted to Nucl. Instr. and Meth. A.

[10] D. Sober et al., Nucl. Instr. and Meth. A 343, 263 (2000).

[11] P.L. Cole, "The IPN-Orsay/UTEP Instrumented Collimator," August (1999) 17pp. unpublished. A. Puga, M.S. thesis, University of Texas at El Paso, "Calibration of the UTEP/Orsay Instrumented Collimator via the LabVIEW-Benchtop Data Acquisition System," December, 2001.

[12] A. Natter, ANB code, URL: http://www.pit.physik.unituebingen.de $\mathrm{X} / \sim$ natter/software/brems-anb.html

[13] K. Schilling et al., Nucl. Phys. B 15, 397 (1970).

[14] Q. Zhao, University of Surrey, Qiang.Zhao@surrey.ac.uk, personal correspondence.

[15] The complete angular distribution for this reaction is given by $W(\cos \theta, \phi, \Phi)=W^{0}\left(\cos \theta, \phi, \rho_{\alpha \beta}^{0}\right)$ $P_{\gamma} \cos 2 \Phi W^{1}\left(\cos \theta, \phi, \rho_{\alpha \beta}^{1}\right)$ $P_{\gamma} \cos 2 \Phi W^{2}\left(\cos \theta, \phi, \rho_{\alpha \beta}^{2}\right)[13]$.

[16] S. Janssen, University of Ghent, stijn.janssen@ ugent.be, personal correspondence.

[17] G. Knochlein, D. Dreschel, and L. Tiator, Z. Phys. C 63, 37 (1994).

[18] SAPHIR Collaboration: M.Q. Tran et al., Phys. Lett. B 445, 20 (1998). 